\title{
A prognostic nomogram for women with primary ovarian signet- ring cell carcinoma
}

\author{
Xijuan Wang ${ }^{1}$, Xiurong $\mathrm{Ke}^{2}$, Junxia Min ${ }^{3}$ \\ ${ }^{1}$ Cancer Institute (Key Laboratory of Cancer Prevention and Intervention, China National Ministry of Education, Key Laboratory of Molecular \\ Biology in Medical Sciences), The Second Affiliated Hospital, Zhejiang University School of Medicine, Hangzhou, China; ${ }^{2}$ Department of Orthopedic \\ Surgery, The Third Hospital Affiliated to Wenzhou Medical University, Rui'an, China; ${ }^{3}$ Division of Hepatobiliary and Pancreatic Surgery, Department \\ of Surgery, The First Affiliated Hospital, Institute of Translational Medicine, Zhejiang University School of Medicine, Hangzhou, China \\ Contributions: (I) Conception and design: All authors; (II) Administrative support: X Ke, J Min; (III) Provision of study materials or patients: X Wang, \\ X Ke; (IV) Collection and assembly of data: X Wang, X Ke; (V) Data analysis and interpretation: All authors; (VI) Manuscript writing: All authors; (VII) \\ Final approval of manuscript: All authors. \\ Correspondence to: Prof. Junxia Min, MD, PhD. Division of Hepatobiliary and Pancreatic Surgery, Department of Surgery, The First Affiliated \\ Hospital, Institute of Translational Medicine, Zhejiang University School of Medicine, Yuhangtang Road 866, Hangzhou 310058, China. \\ Email: junxiamin@zju.edu.cn.
}

Background: Primary ovarian signet-ring cell carcinoma (POSRCC) is a rare subtype of ovarian carcinoma that is characterized by abundant mucin accumulation. POSRCC is aggressive, and the prognostic factors associated with its clinical outcome remain poorly defined. This study aimed to elucidate the clinical characteristics and survival of patients with POSRCC, and to establish an effective prognostic nomogram and risk stratification model to predict the risks associated with patient outcomes.

Methods: Data of patients with POSRCC from the period 1975 to 2016 were collected from the Surveillance, Epidemiology, and End Results (SEER) database. Univariable and multivariable analyses of demographic factors, clinicopathological characteristics, and treatments were conducted to identify significant prognostic parameters. The identified independent variables were integrated to develop a nomogram and risk stratification model. The discrimination and calibration of the nomogram were assessed with the concordance index (C-index), receiver operating characteristic (ROC) curves, and calibration curves. Results: A total of 172 patients were identified as being eligible to participate in this study. The median overall survival (OS) time was 7 months [95\% confidence interval (CI), 4.6-9.4 months]. The 1-, 3-, and 5 -year OS rates were $35.5 \%, 15.3 \%$, and $6 \%$, respectively. A multivariable analysis of the primary patients identified the independent predictors for survival as age at diagnosis, race, marital status, $\mathrm{T}$ (primary tumor size) stage, and chemotherapy, which were all incorporated into the nomogram. The C-index was 0.70 (95\% CI, 0.66-0.75), which was statistically higher than that of the International Federation of Gynecology and Obstetrics (FIGO) staging system (0.58; 95\% CI, 0.53-0.63). ROC curve analysis also showed that the nomogram had good discrimination, with an area under the curve (AUC) of 0.74, 0.62, and 0.71 for 1-, 3-, and 5-year survival, respectively. The calibration curves showed good agreement between the prediction by the nomogram and actual observations. A risk stratification model was further used to classify patients into a low-risk or high-risk group. The median OS time for the low- and high-risk groups was 13.0 months (95\% CI, 9.33-16.67) and 2.0 months (95\% CI, 1.12-2.89), respectively. Surgery did not significantly prolong survival in either group [low-risk group: hazard ratio (HR), 0.69; 95\% CI, 0.45-1.07; P=0.09; high-risk group: HR, 0.55; 95\% CI, 0.46-0.67; P=0.18].

Conclusions: The proposed nomogram and risk stratification model showed accurate prognostic prediction for POSRCC. These methods could improve individualized evaluations of survival and therapeutic decisions for patients with POSRCC.

Keywords: Chemotherapy; nomogram; ovarian cancer; Surveillance, Epidemiology, and End Results database (SEER database); survival 
Submitted Sep 04, 2020. Accepted for publication Dec 18, 2020.

doi: $10.21037 /$ atm-20-6280

View this article at: http://dx.doi.org/10.21037/atm-20-6280

\section{Introduction}

Signet-ring cell carcinoma (SRCC) is a subtype of adenocarcinoma that is characterized by abundant mucin accumulation in tumor cells individually or in small groups. Most SRCCs of the ovary are metastatic, originating primarily from the gastrointestinal tract, and occasionally from other organs, such as the breast, pancreaticobiliary tract, urinary bladder, cervix, and renal pelvis (1). However, in rare instances, SRCCs arise as a primary ovarian tumor. To date, very few cases of primary ovarian SRCC (POSRCC) have been reported (1-9). The signet-ring cells in POSRCC can coexist with mucinous carcinoma (1-9) or papillary serous carcinoma (3). Generally, surgery and adjuvant systemic chemotherapy are used to treat POSRCC $(4,9)$. However, the treatment outcomes of POSRCC are generally poor, and vary widely among published reports (1-9). The current classifications for ovarian cancer of the International Federation of Gynecology and Obstetrics (FIGO) and the World Health Organization (WHO) have improved clinicians' ability to predict the prognosis of patients; however, these classifications still have deficiencies (10). Given that the clinical characteristics and survival of POSRCC have yet to be defined explicitly, it is rational to use a population-based cancer database to elucidate them.

In recent years, nomograms have been shown to be a viable statistical predictive model for assessing clinical outcomes and have been developed for numerous types of cancer (11-16). Nomograms can achieve improved accuracy in the prediction of prognostic outcomes compared to many traditional staging systems $(11-13,16)$. To the best of our knowledge, the survival of patients with POSRCC has yet to be systematically studied. Thus, by creating and validating a proposed nomogram based on the large and widely acknowledged Surveillance, Epidemiology, and End Results (SEER) database, we sought to define a broadly applicable and generalizable nomogram and risk stratification model for patients with POSRCC. This study also evaluated whether the proposed nomogram could predict survival more accurately than the currently available staging system.

We present the following article in accordance with the STROBE reporting checklist (available at http://dx.doi. org/10.21037/atm-20-6280).

\section{Methods}

The study was conducted in accordance with the Helsinki Declaration (as revised in 2013). POSRCC data from 1975 to 2016 were retrieved from the latest version of the SEER database, as released in November 2018, using the SEER*Stat software (version 8.3.6). The SEER program contains cancer incidence data from 18 population-based cancer registries covering approximately $34.6 \%$ of the United States' population. As the data in the SEER database have been de-identified and coded for public use, the present study was exempt from the requirement to obtain local institutional review board approval.

The diagnosis of POSRCC was identified using the International Classification of Disease for Oncology, Third Edition (ICD-O-3) histology codes for SRCC (8490/3), behavior recode for analysis (malignant), sequence number (one primary only), and primary site label (C56.9-Ovary). The diagnoses were all microscopically confirmed, and the data for cases that were first identified at autopsy or only at death were excluded from the study.

Variables included demographic information (e.g., race, age at diagnosis, and marital status), tumor characteristics [e.g., primary site, tumor-node-metastasis (TNM) stage, and laterality], treatment (e.g., surgery and chemotherapy), and active follow-up for survival (survival months and vital status). In the SEER database, race is coded as white, black, or other (e.g., American Indian/Alaskan native or Asian/Pacific Islander). Marital status is coded as single, married, or divided. Divided status includes individuals who have been divorced, separated or widowed. Between 1975 and 2016, patients were categorized according to three different staging systems (the $3^{\text {rd }}, 6^{\text {th }}$, and $7^{\text {th }}$ editions of the TNM classification). Overall survival (OS) was defined as the time interval from diagnosis to death from any cause or last follow-up. Due to the potential risk of selection bias induced by the large time span, the data were further divided into two time periods for further analysis.

\section{Statistical analysis}

The Kaplan-Meier method was used to generate survival plots. The log-rank test was then used to compare 


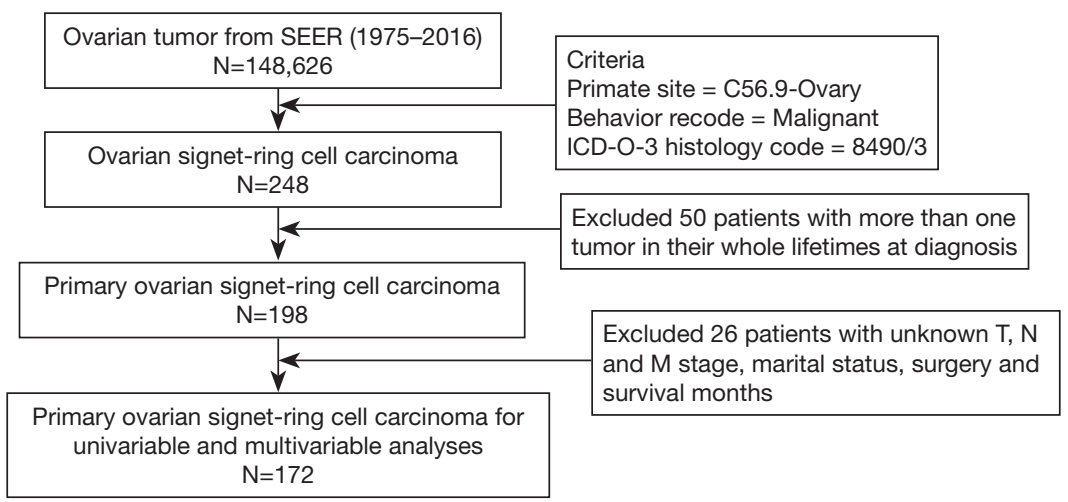

Figure 1 Patient selection flowchart. SEER, Surveillance, Epidemiology, and End Result Program; ICD-O-3, International Classification of Disease for Oncology, Third Edition.

groups. The Cox proportional hazards regression model was used in univariable and multivariable analyses to identify independent predictors of OS in patients with POSRCC. Based on significant prognostic factors in the multivariable analysis, a nomogram was constructed to predict OS probability at 1,3 , and 5 years. The nomogram was subjected to validation through evaluation of its discrimination ability and calibration (17). Discrimination is a measure of the probability of concordance between observed and predicted outcomes in Harrell C-statistic and receiver operating characteristic (ROC) analyses. The higher the concordance index (C-index), the more accurate the prognostic prediction of patient outcomes (12). ROC curve analysis was performed to estimate the area under the curve (AUC). To assess the relationship between the predicted probabilities and the frequency of the observed outcomes, calibration plots were generated using 1,000 bootstrap resampling replicates of the study. All statistical analyses were performed using IBM SPSS statistics version 23.0 (SPSS, Chicago, IL, USA), and R statistical software version 3.6.3 (R Foundation for Statistical Computing, Vienna, Austria). All $\mathrm{P}$ values were two-sided, and a $\mathrm{P}$ value of 0.05 was used as the threshold to determine statistical significance.

\section{Results}

Figure 1 summarizes the process for patient selection. Among 148,626 patients with an ovarian tumor, 248 (0.17\%) patients were identified as having ovarian SRCC using the ICO-O-3 histology code (8490/3) and primary tumor site labeled as ovary (C56.9). Of them, 198 (0.13\%) POSRCC patients who had only one type of primary tumor in their entire lifetime at the time of diagnosis were validated. Twenty-six patients were excluded as their data included unknown variables. Thus, 172 eligible patients from the period 1990 to 2016 were included in the present study (Figure 1).

Table 1 summarizes the baseline clinical characteristics and survival of the POSRCC patients. The median age at diagnosis was 64 years (interquartile range: 53-75 years), which is consistent with previous reports (9). In relation to race, most patients were white $(82.6 \%)$, and black patients and patients of other races each accounted for $8.7 \%$ of the study population. A majority of POSRCCs in the study were unilateral (70.3\%), with unilaterality being widely accepted as a feature indicative of primary ovarian origin (18). One-half of the cases were diagnosed with definite distant metastasis. Nearly $90 \%$ of POSRCC patients were diagnosed at advanced stages. Chemotherapy was received by 106 patients $(61.6 \%)$, and 83 patients $(48.3 \%)$ were treated with surgery. A total of 58 patients (33.7\%) received both chemotherapy and surgery. In this study, the results showed that combined treatment with surgery and chemotherapy yielded superior OS compared to chemotherapy alone or surgery alone (median OS, 16 vs. 9 vs. 2 months, log-rank $\mathrm{P}<0.001)$.

The median OS time for all patients was 7 months [95\% confidence interval (CI), 4.6-9.4 months], and there were 157 (91.3\%) deaths, including 95.5\% cancer-specific deaths by the end of last follow-up. The 1-, 3-, and 5-year OS rates were $35.5 \%, 15.3 \%$, and $6 \%$, respectively. KaplanMeier survival analyses, and univariable and multivariable analyses were undertaken to identify the significant prognostic predictors in the study (Figure 2 and Table 2, respectively). Age at diagnosis, race, $\mathrm{T}$ (primary tumor 
Table 1 Baseline clinical characteristics and survival rates of POSRCC patients from the SEER database $(\mathrm{n}=172)$

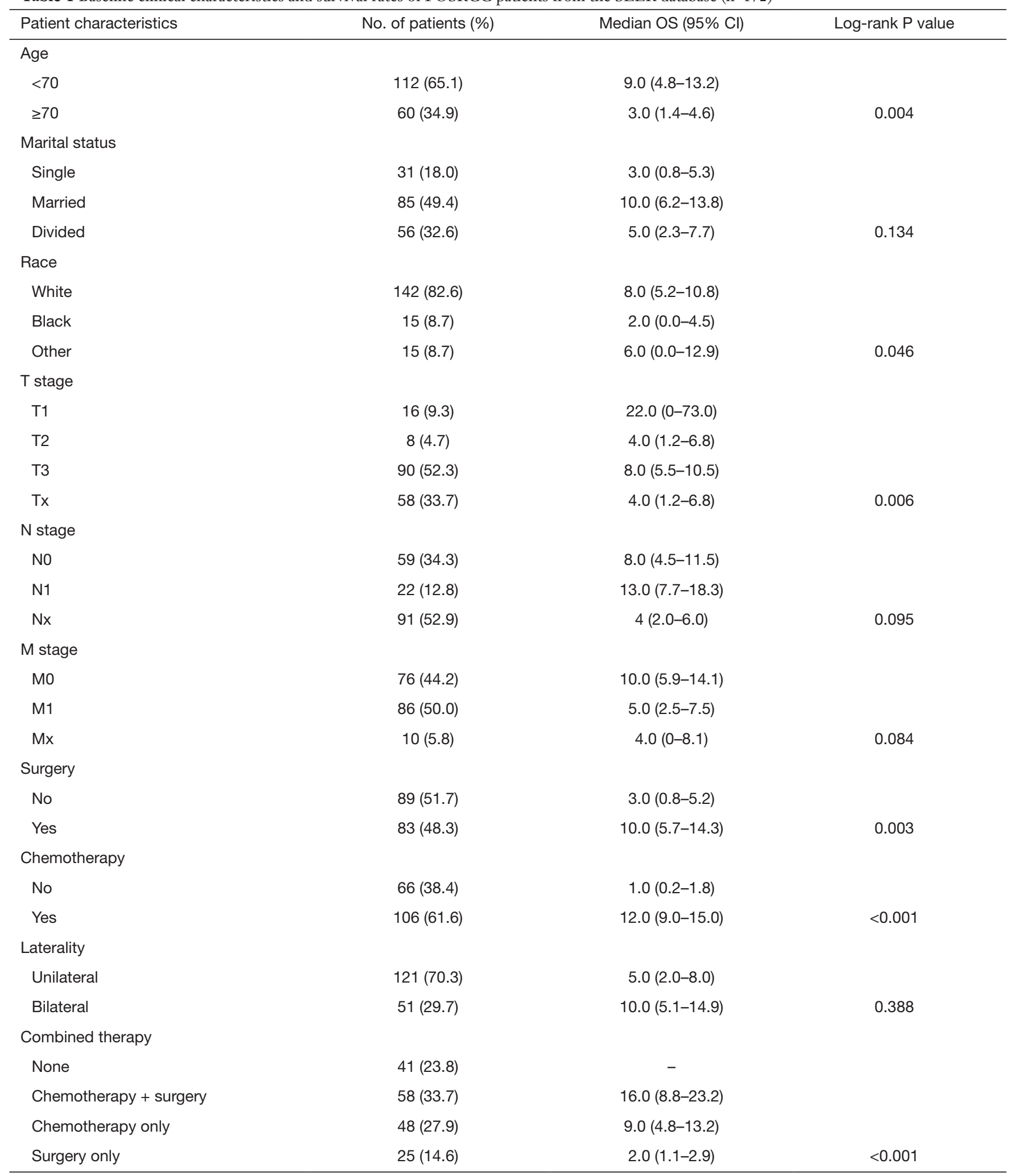

Table 1 (continued) 
Table 1 (continued)

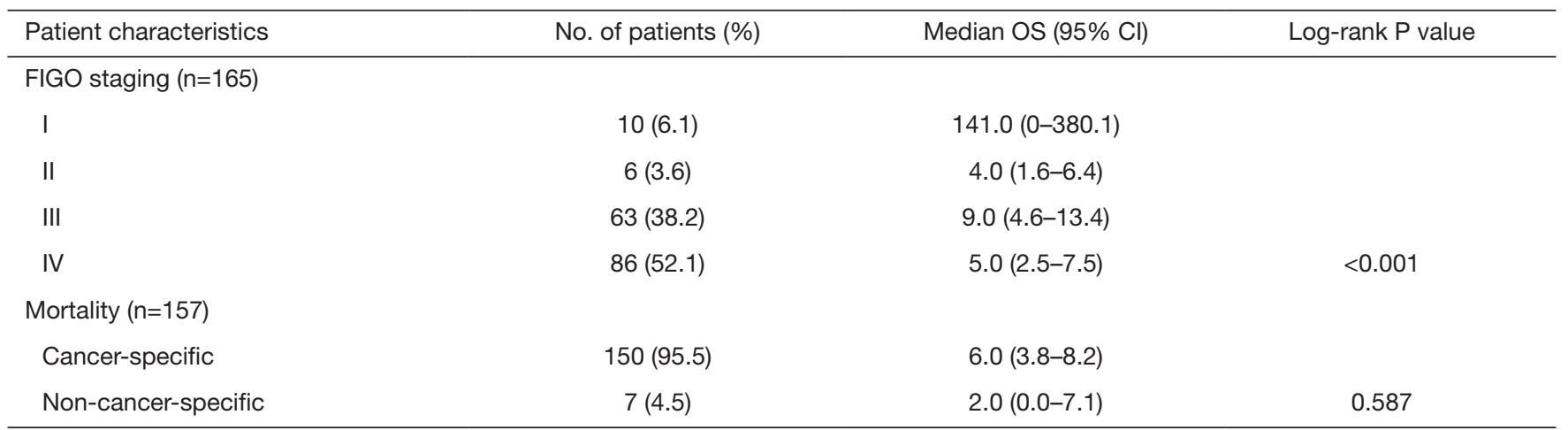

POSRCC, primary ovarian signet-ring cell carcinoma; SEER, Surveillance, Epidemiology, and End Results; OS, overall survival; CI, confidence interval; T, primary tumor size; N, regional lymph nodes; M, distant metastasis; FIGO, International Federation of Gynecology and Obstetrics.
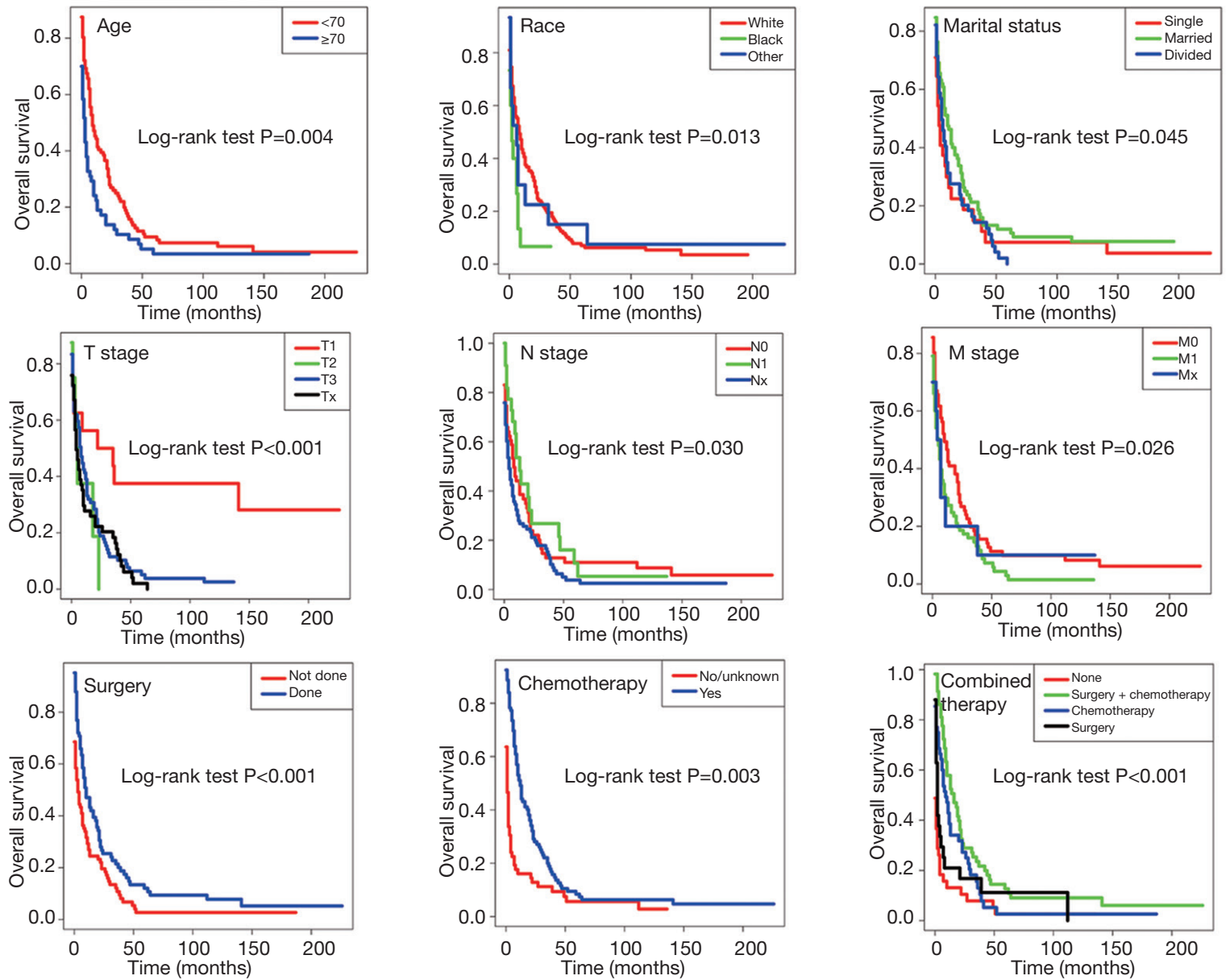

Figure 2 Kaplan-Meier curves with log-rank tests for OS. OS, overall survival. 
Table 2 Univariable and multivariable analyses for OS

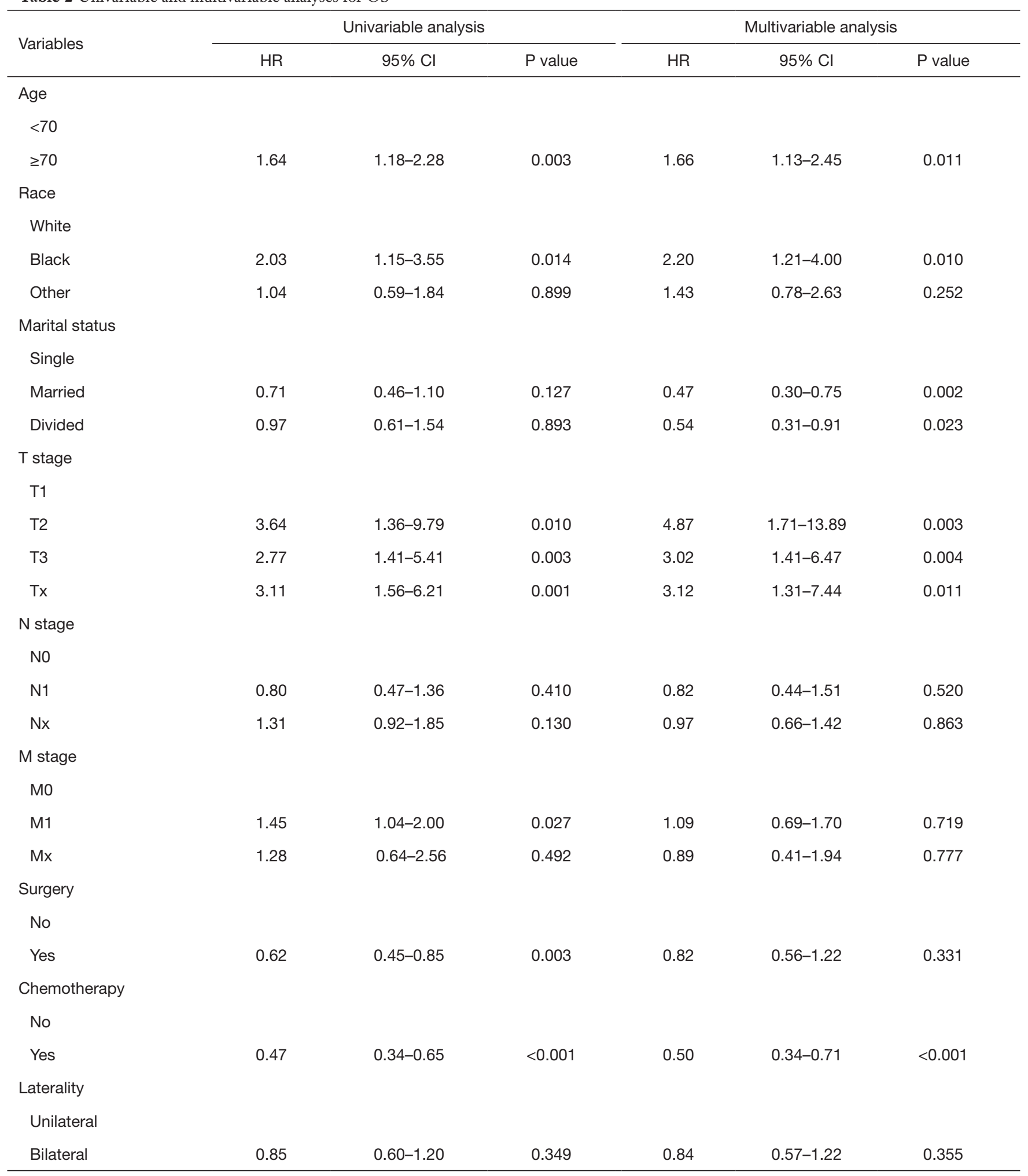

OS, overall survival; HR, hazard ratio; $\mathrm{Cl}$, confidence interval; T, primary tumor size; $\mathrm{N}$, regional lymph nodes; $\mathrm{M}$, distant metastasis. 


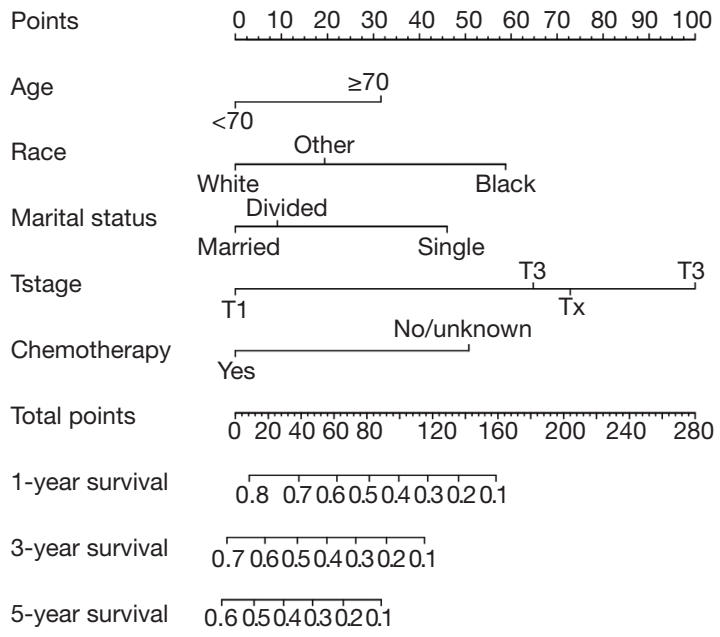

Figure 3 Nomogram for predicting 1-, 3-, and 5-year OS in POSRCC patients. Instructions for using the nomogram: Locate the patient's characteristics on each variable axis. Draw a straight line upward to the 'points' scale to determine the score for each variable. Locate the sum of these scores on the 'total points' axis. Draw a straight line downwards to the survival axes to determine the probability of 1-, 3- and 5-year OS. OS, overall survival; POSRCC, primary ovarian signet-ring cell carcinoma.

size) stage, and chemotherapy were significantly associated with OS in both the univariable and multivariable analyses. Marital status was also identified as a significant prognostic predictor in the multivariable analysis. The multivariable analysis showed that patients diagnosed with POSRCC at an older age were more likely to die of ovarian cancer, with a hazard ratio (HR) of 1.66 (95\% CI, 1.13-2.45; $\mathrm{P}=0.011$ ). White patients had better survival than black patients (HR, 2.20; 95\% CI, 1.21-4.00; P=0.010) patients. Single patients had poorer OS than married patients (HR, 0.47; 95\% CI, 0.30-0.75; $\mathrm{P}=0.002)$ and patients who had been married before (HR, 0.54; 95\% CI, 0.31-0.91; $\mathrm{P}=0.023$ ). More advanced $\mathrm{T}$ stages were also found to be associated with worse OS (T2: HR, 4.87; 95\% CI, 1.71-13.89; $\mathrm{P}=0.003$; T3: HR, 3.02; 95\% CI, 1.41-6.47; $\mathrm{P}=0.004)$. No significant difference was found in the OS for unilateral and bilateral POSRCCs. The OS of patients who received chemotherapy (HR, 0.50; 95\% CI, 0.34-0.71; $\mathrm{P}<0.001$ ) was significantly better than that of patients who did not receive chemotherapy. The univariable analysis showed that patients who received surgery generally had better OS than their counterparts who did not receive surgery. However, after adjusting for clinically relevant factors, surgery was found to have no significant association with patients' OS.

All the prognostic predictors that were found to have a significant association with OS in the multivariable analysis were integrated into the construction of the nomogram (Figure 3). The C-index for OS prediction was 0.70 (95\% CI, 0.66-0.75). The nomogram demonstrated better discrimination for predicting OS than the FIGO staging system, which had a C-index of 0.58 (95\% CI, 0.53-0.63). The nomogram also exhibited good discrimination in the ROC analysis, with AUCs of 0.74, 0.62, and 0.71 for 1-, 3-, and 5-year survival, respectively (Figure 4). The calibration curves for the probability of survival at 1,3 , and 5 years showed good agreement between the prediction by the nomogram and actual observations (Figure 5). The results suggested that the nomogram could be used to accurately predict the survival of patients with POSRCC.

To further examine the risk of selection bias, the time spanning 1990-2016 was divided into two periods: 1990 to 2002 and 2003 to 2016. There were 87 POSRCC patients in the 1990 to 2002 group, and 85 patients in the 2003 to 2016 group. Nomograms were plotted based on the data from the two periods (Figure S1), which were similar to the nomogram based on the whole data of the 172 POSRCC patients (Figure 3). The C-index for the prediction of OS was 0.71 (95\% CI, 0.71-0.72) in the 1990 to 2002 group, and 0.72 (95\% CI, $0.72-0.73$ ) in the 2003 to 2016 group. The ROC analyses showed that the nomograms had good discrimination ability, with AUCs for 1-, 3-, and 5-year survival of $0.7,0.66$, and 0.89 , respectively, in the 1990 to 2002 group (Figure S2A,B,C), and 0.8, 0.61, and 0.57, respectively, in the 2003 to 2016 group (Figure S2D,E,F). In both groups, the calibration curves for the probability of survival at 1,3 , and 5 years demonstrated good agreement between the prediction by the nomograms and actual observations (Figure S3). Thus, the results showed that the large time span had very little effect on the risk of selection bias.

Additionally, POSRCC patients could be distinctly stratified into low-risk (54.7\%) and high-risk (45.3\%) groups according to their predicted risk. The median OS time in the low- and high-risk groups was 13.0 months (95\% CI, 9.33-16.67), and 2.0 months (95\% CI, 1.12-2.89), respectively. The Kaplan-Meier curve indicated that the risk stratification model could accurately differentiate survival in the two prognostic groups (see Figure $6 A$ ).

To further assess the survival benefits associated with 

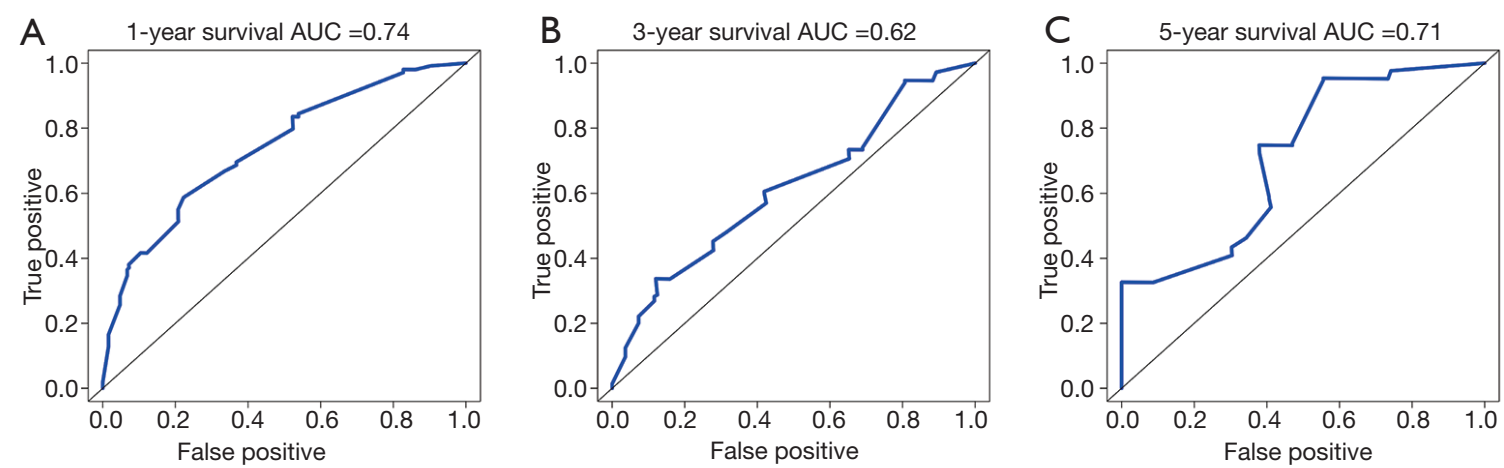

Figure 4 ROC curve analyses with estimated AUC for (A) 1-, (B) 3-, and (C) 5-year OS. ROC, receiver operating characteristic; AUC, area under the curve; OS, overall survival.

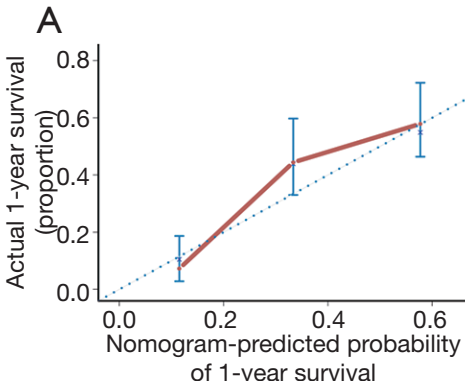

of 1-year survival

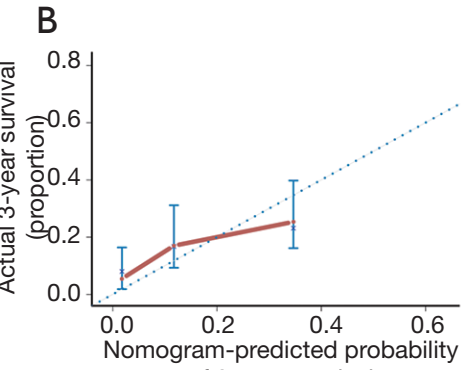

of 3-year survival

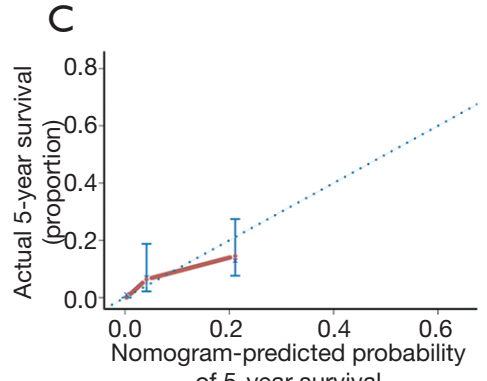

of 5-year survival

Figure 5 Calibration curves for predicting OS at (A) 1-, (B) 3-, and (C) 5-year. Nomogram-predicted probability of OS is plotted on the $\mathrm{X}$-axis; actual OS is plotted on the Y-axis. The red line represents OS while the blue line represents $95 \%$ CIs. OS, overall survival; CI, confidence interval.
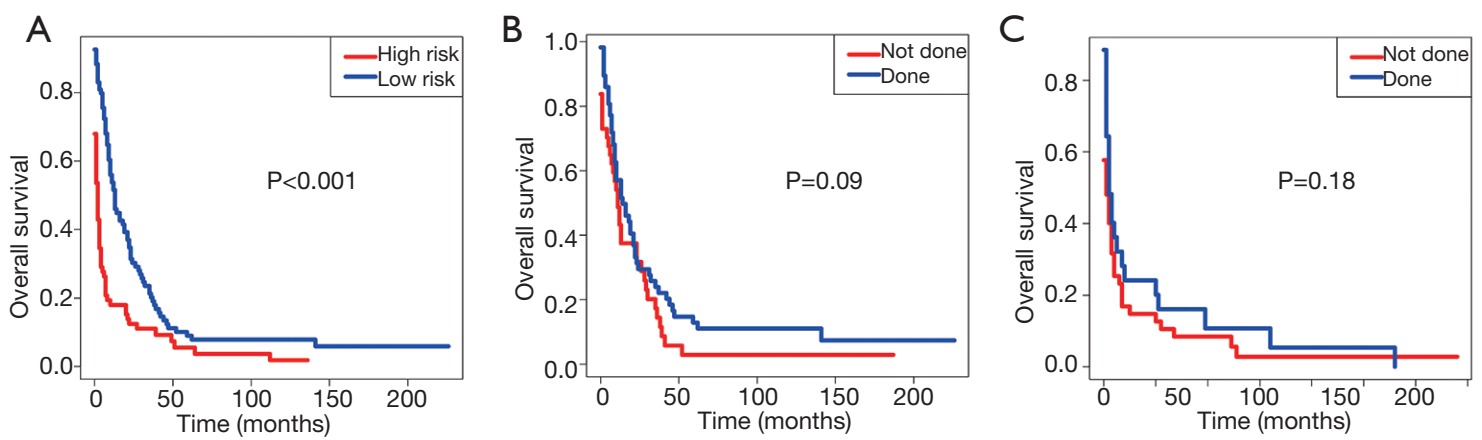

Figure 6 Kaplan-Meier curve of the low- and high-risk groups (A), and survival benefit of surgery in the low-risk (B) and high-risk (C) groups.

surgery, Kaplan-Meier curves were generated for the stratified risk groups. The results demonstrated that surgery did not significantly prolong OS in either group (low-risk group: $\mathrm{HR}, 0.69 ; 95 \% \mathrm{CI}, 0.45-1.07 ; \mathrm{P}=0.09$; high-risk group: HR, 0.55; 95\% CI, 0.46-0.67, $\mathrm{P}=0.18$ ) (Figure 6B,C).

\section{Discussion}

\section{Principal findings}

Due to the rarity of POSRCC as a clinical entity, the analysis of the clinical characteristics of this disease and the 
creation of models to predict prognosis are of profound significance. In the study, we comprehensively elucidated the clinical characteristics and survival of patients with POSRCC, and developed a nomogram to predict survival outcomes. We demonstrated that variables, such as white race, marriage, and chemotherapy, were independent protective factors, while increasing age and advanced $\mathrm{T}$ stages were independently associated with worse OS. Further discrimination and calibration indicated that the nomogram had optimal predictive accuracy. Notably, in our established risk stratification model, neither the low-risk group nor the high-risk group showed improved survival with surgery, suggesting that surgery may not be a strong risk factor in the prognosis of POSRCC.

\section{Results}

The POSRCC patient sample size in the current study was much larger than those used in previous studies (1-9). In this study, we found that the prognosis for POSRCC patients was extremely poor, with a median OS time of 7 months. POSRCC tended to occur at an advanced age (the patients in the present study had a median age of 64 years). Similar to the findings of another study, the overwhelming majority of POSRCC patients were white. Nearly $90 \%$ of POSRCC patients presented at advanced stages (3). Patients who presented in the early stages had a favorable prognosis $(1,5,7,9)$, but such patients represented only a very small proportion of the sample in this study.

Adjuvant chemotherapy is usually considered for patients with completely resected ovarian tumors who are at an early stage (19). Our study showed that POSRCC patients were more commonly treated with chemotherapy than with surgery. Further, POSRCC patients who underwent chemotherapy had a significantly better prognosis than those who did not undergo chemotherapy (median OS, 12 vs. 1 month; log-rank $\mathrm{P}<0.001)$. This effect was verified by the multivariable Cox regression, which showed that chemotherapy was a strong protective predictor with a HR of 0.50 (95\% CI, 0.34-0.71; $\mathrm{P}<0.001)$. The univariable analysis showed that surgery was significantly associated with OS; however, this effect disappeared in the multivariable analysis. The risk stratification model was able to accurately classify patients into low- or highrisk group. Under this model, the median OS time for the low-risk and high-risk groups was 13.0 and 2.0 months, respectively.

\section{Clinical implications}

To the best of our knowledge, this appears to be the first large-sample-size retrospective study to develop a predictive nomogram for the prognosis of POSRCC patients. The predictive methods in this study may help to predict which individual patients are at a high risk of poor prognosis, and may improve the clinical decisions made by clinical physicians. In addition, the established risk stratification system differentiates high-risk patients who need more adjuvant therapies, and such patients should be encouraged to participate in clinical trials of novel drugs and treatment strategies.

\section{Research implications}

In this study, surgery was not found to be a strong risk factor; however, this study could not specifically examine different surgery types, and the results approached significance. Thus, further clinical trials with large sample sizes should be conducted to validate the operation indications for POSRCC patients. Chemotherapy was identified as an independent prognostic factor; thus, the establishment of a concrete and optimal chemotherapy regimen will be crucial in improving the prognosis of POSRCC patients.

\section{Strengths and limitations}

This study has a number of strengths. First, our nomogram produced a C-index of 0.70, which was higher than that of the FIGO classification system, indicating the nomogram to have better ability to predict survival. Second, the nomogram includes demographic indices, such as marriage, which are not been included as variables in the FIGO staging system. Marriage has been suggested to be an independent prognostic factor for ovarian cancer $(20,21)$. In the present study, marital status had a strong association with OS after adjustment in the multivariable analysis. Marital status has not been included in traditional staging systems; however, recently, it has been found to play a role in increasing the predictive performance in relation to various tumors $(22,23)$.

This study also has several limitations. First, the main population recruited in the SEER database comprises white patients from the United States, whose conditions, such as their living environments, climate conditions, and eating habits, differ to those of other races around 
the world. Further research needs to be conducted to determine whether the conclusions drawn from the SEER database are applicable to other races. Second, marital status in the SEER database is only recorded at the time of diagnosis. There was no data as to whether patients' subsequent marital status had changed at the time of the follow-up visits or in any other records. Thus, the effects of such changes on the study cannot be estimated. Third, information about the chemotherapy regimens and the sequence of chemotherapy and surgery was not available in the SEER database, making the effectiveness of neoadjuvant chemotherapy unevaluable. Finally, it should be noted that this study was retrospective in nature, which may have led to selection bias. Rigorous validation methods (e.g., bootstrapped calibration and bias-corrected estimates) were used to validate the nomogram; however, further studies should seek to verify the effectiveness of the nomogram's clinical application.

As POSRCC is rare and aggressive, doctors should be aware of this disease. To improve the early diagnosis rate of POSRCC, it is imperative that understandings of the differential diagnosis of POSRCC should be improved. Further research should seek to make the differential diagnosis of POSRCC easier and more precise, and to improve the prognosis of this disease.

\section{Conclusions}

A predictive nomogram and risk stratification model were generated to predict the individual survival rates of POSRCC patients. These methods may assist clinical physicians to evaluate the risk of POSRCC among patients and make more informed decisions in relation to individual treatment.

\section{Acknowledgments}

We acknowledge the efforts of the SEER program tumor registries in creating the SEER database (https://seer. cancer.gov/).

Funding: This study was supported in part by grants from the Medical and Health Research Project of Zhejiang Province (2020KY929) and the Basic Medical Technology Project of Wenzhou (Y20180307).

\section{Footnote}

Reporting Checklist: The authors have completed the
STROBE reporting checklist. Available at http://dx.doi. org/10.21037/atm-20-6280

Conflicts of Interest: All authors have completed the ICMJE uniform disclosure form (available at http://dx.doi. org/10.21037/atm-20-6280). The authors have no conflicts of interest to declare.

Ethical Statement: The authors are accountable for all aspects of the work, including ensuring that questions related to the accuracy or integrity of any part of the work have been appropriately investigated and resolved. The study was conducted in accordance with the Helsinki Declaration (as revised in 2013).

Open Access Statement: This is an Open Access article distributed in accordance with the Creative Commons Attribution-NonCommercial-NoDerivs 4.0 International License (CC BY-NC-ND 4.0), which permits the noncommercial replication and distribution of the article with the strict proviso that no changes or edits are made and the original work is properly cited (including links to both the formal publication through the relevant DOI and the license). See: https://creativecommons.org/licenses/by-nc-nd/4.0/.

\section{References}

1. McCluggage WG, Young RH. Primary ovarian mucinous tumors with signet ring cells: report of 3 cases with discussion of so-called primary Krukenberg tumor. Am J Surg Pathol 2008;32:1373-9.

2. Schiller W, Kozoll DD. Primary signet-ring cell carcinoma of the ovary. Am J Obstet Gynecol 1941;41:70-8.

3. Che M, Tornos C, Deavers MT, et al. Ovarian mixedepithelial carcinomas with a microcystic pattern and signet-ring cells. Int J Gynecol Pathol 2001;20:323-8.

4. El-Safadi S, Stahl U, Tinneberg HR, et al. Primary signet ring cell mucinous ovarian carcinoma: a case report and literature review. Case Rep Oncol 2010;3:451-7.

5. Jaya Ganesh P, Vimal Chander R, Kanchana MP, et al. Primary ovarian mucinous carcinoma with signet ring cells - report of a rare case. J Clin Diagn Res 2014;8:FD12-3.

6. Tayal U, Bajpai M, Jain A. Primary signet ring carcinoma of ovary case report. Rama Univ J Med Sci 2015;1:38-41

7. Kim JH, Cha HJ, Kim KR, et al. Primary ovarian signet ring cell carcinoma: a rare case report. Mol Clin Oncol 2018;9:211-4.

8. Jiao Y, Lu B. Poorly differentiated mucinous carcinoma 
with signet ring cells in an ovarian endometriotic cyst: a case report. Diagn Pathol 2019;14:73.

9. Pongsuvareeyakul T, Charoenkwan K, Suprasert P, et al. Primary signet ring cell carcinoma with neuroendocrine differentiation arising in mucinous borderline tumor of the ovary. Gynecol Oncol Rep 2019;31:100522.

10. Gomes Ferreira M, Sancho de Salas M, Gonzalez Sarmiento R, et al. Changes in the management and prognosis of ovarian cancer due to the new FIGO and WHO classifications: a case series observational descriptive study. Seven years of follow-up. Int J Gynecol Cancer 2018;28:1461-70.

11. Han DS, Suh YS, Kong SH, et al. Nomogram predicting long-term survival after $\mathrm{d} 2$ gastrectomy for gastric cancer. J Clin Oncol 2012;30:3834-40.

12. Wang Y, Li J, Xia Y, et al. Prognostic nomogram for intrahepatic cholangiocarcinoma after partial hepatectomy. J Clin Oncol 2013;31:1188-95.

13. Yang Y, Zhang YJ, Zhu Y, et al. Prognostic nomogram for overall survival in previously untreated patients with extranodal NK/T-cell lymphoma, nasal-type: a multicenter study. Leukemia 2015;29:1571-7.

14. Fakhry C, Zhang Q, Nguyen-Tân PF, et al. Development and validation of nomograms predictive of overall and progression-free survival in patients with oropharyngeal cancer. J Clin Oncol 2017;35:4057-65.

15. Berardi G, Morise Z, Sposito C. Development of a nomogram to predict outcome after liver resection for hepatocellular carcinoma in Child-Pugh B cirrhosis. J

Cite this article as: Wang $\mathrm{X}, \mathrm{Ke} \mathrm{X}, \mathrm{Min} \mathrm{J}$. A prognostic nomogram for women with primary ovarian signet-ring cell carcinoma. Ann Transl Med 2021;9(7):525. doi: 10.21037/atm20-6280
Hepatol 2020;72:75-84.

16. Wang Z, Wang H, Sun X. A risk stratification model for predicting overall survival and surgical benefit in triplenegative breast cancer patients with de novo distant metastasis. Front Oncol 2020;10:14.

17. Harrell FE Jr, Lee KL, Mark DB. Multivariable prognostic models: issues in developing models, evaluating assumptions and adequacy, and measuring and reducing errors. Stat Med 1996;15:361-87.

18. Leen SLS, Singh N. Pathology of primary and metastatic mucinous ovarian neoplasms. J Clin Pathol 2012;65:591-5.

19. Lawrie TA, Winter-Roach BA, Heus P, et al. Adjuvant (post-surgery) chemotherapy for early stage epithelial ovarian cancer. Cochrane Database Syst Rev 2015;2015:CD004706.

20. Mahdi H, Kumar S, Munkarah AR, et al. Prognostic impact of marital status on survival of women with epithelial ovarian cancer. Psychooncology 2013;22:83-8.

21. Aizer AA, Chen MH, McCarthy EP, et al. Marital status and survival in patients with cancer. J Clin Oncol 2013;31:3869-76.

22. Dong F, Shen Y, Gao F, et al. Nomograms to predict individual prognosis of patients with primary small cell carcinoma of the bladder. J Cancer 2018;9:1152-64.

23. Zhang G, Li Z, Song D, et al. Nomograms to predict individual prognosis of patients with squamous cell carcinoma of the urinary bladder. BMC Cancer 2019;19:1200. 

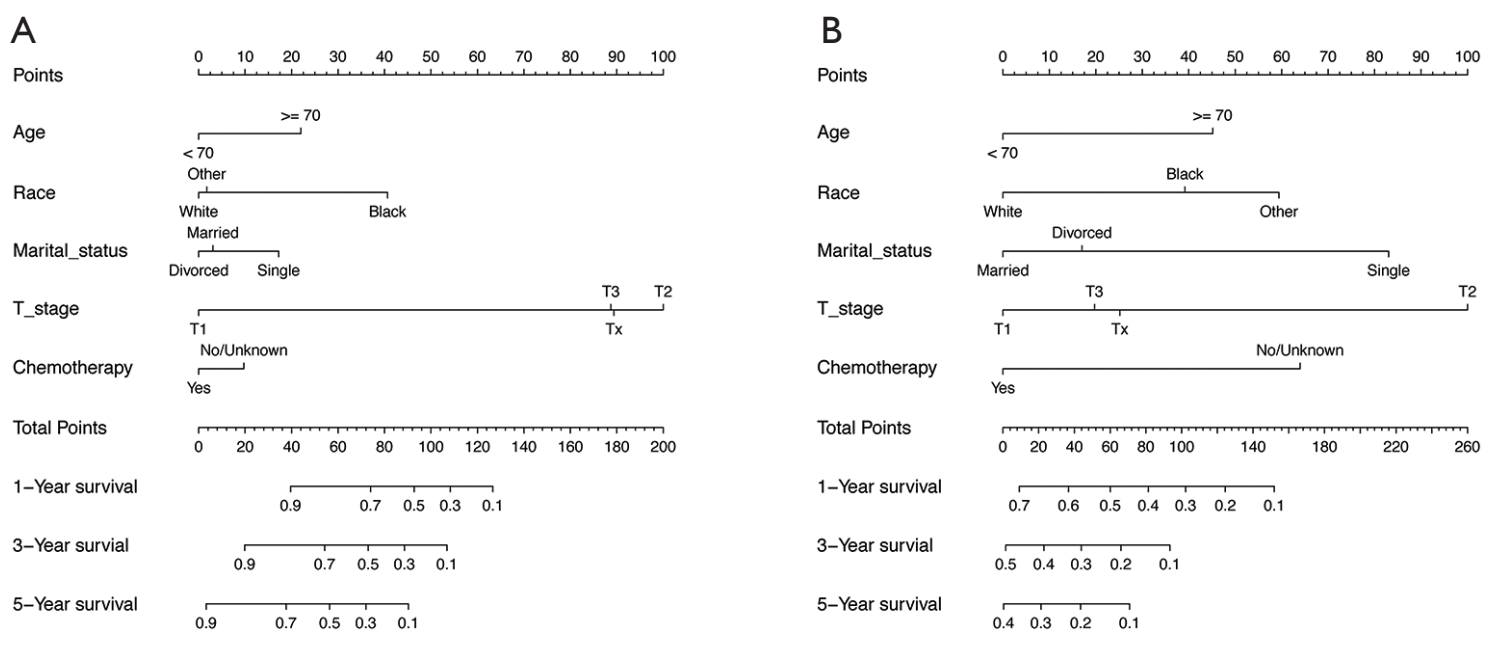

Figure S1 Nomograms for predicting 1-, 3-, and 5-year OS of POSRCC patients from 1990 to 2002 (A) and from 2003 to 2016 (B). OS, overall survival; POSRCC, primary ovarian signet-ring cell carcinoma.
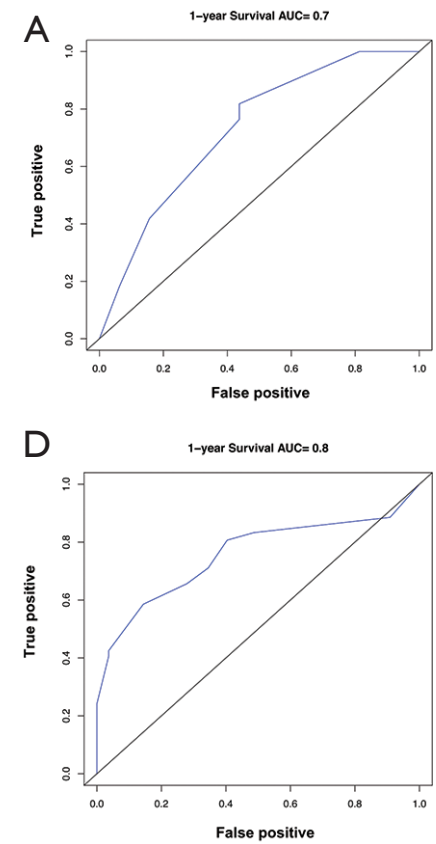
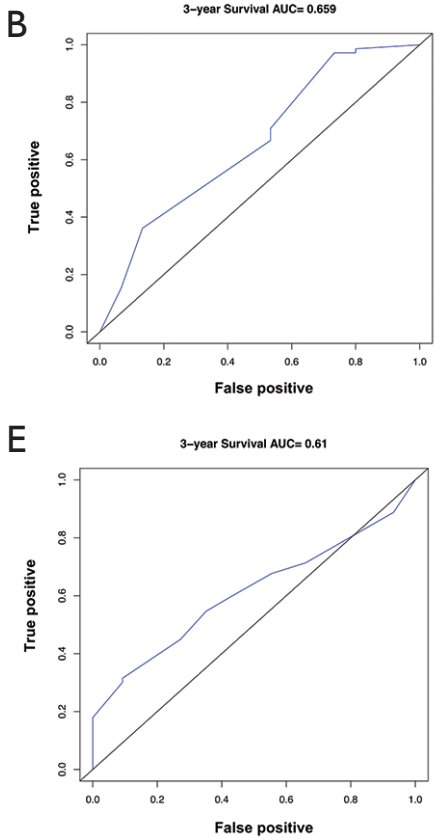

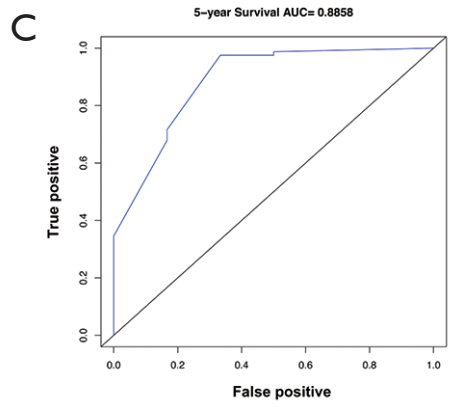

$\mathrm{F}$

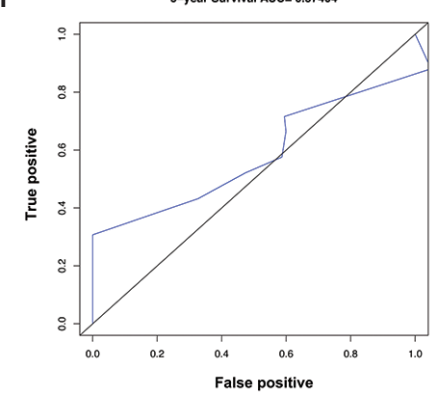

Figure S2 ROC curve analyses with estimated AUCs for 1-, 3-, and 5-year OS of POSRCC patients from 1990 to 2002 (A,B,C) and from 2003 to 2016 (D,E,F). ROC, receiver operating characteristic; AUC, area under the curve; OS, overall survival; POSRCC, primary ovarian signet-ring cell carcinoma.
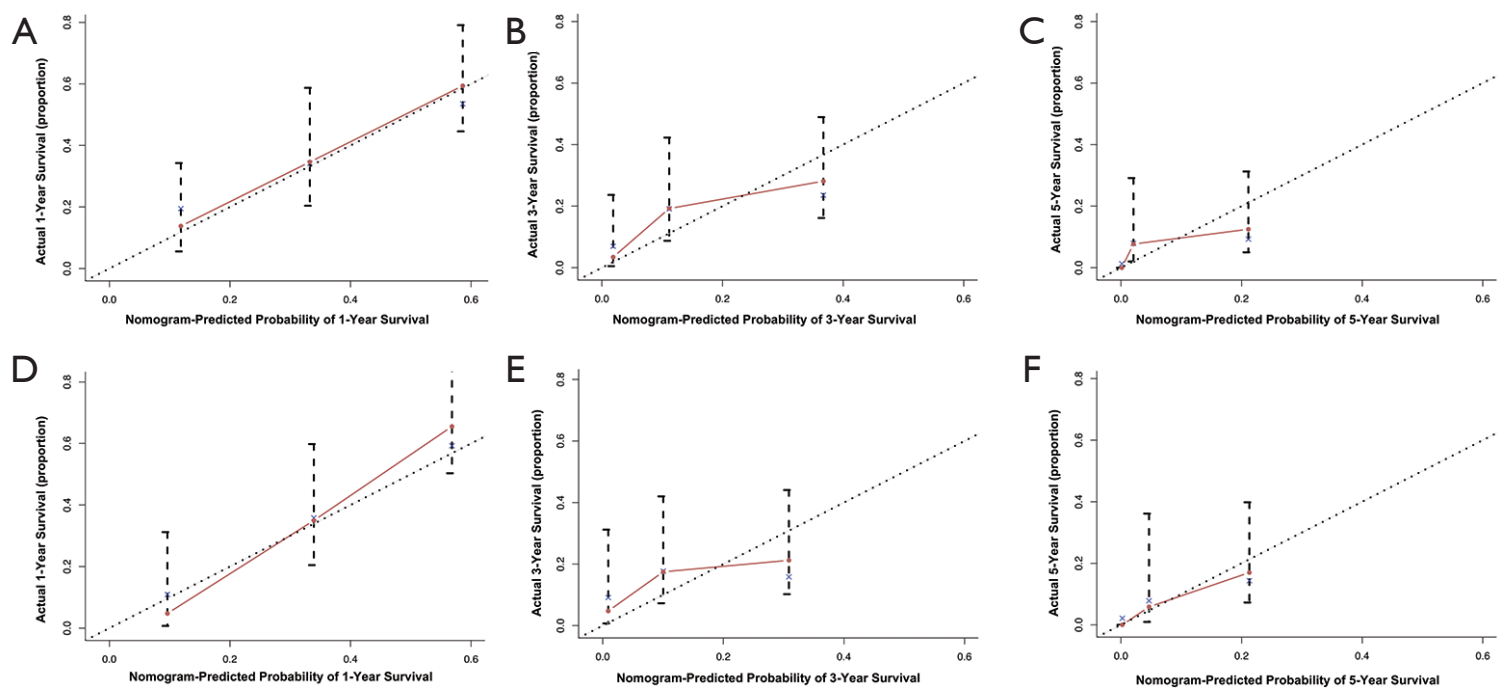

Figure S3 Calibration curves for predicting the OS of POSRCC patients at 1, 3, and 5 years from 1990 to 2002 (A,B,C) and from 2003 to 2016 (D,E,F). OS, overall survival; POSRCC, primary ovarian signet-ring cell carcinoma. 